\title{
Metal-organic frameworks (MOFs) for the adsorptive removal of selected endocrine disrupting compounds (EDCs) from aqueous solution: a review
}

\begin{abstract}
The progress of water-stable metal-organic frameworks (MOFs) has drawn interest from scientists to expand the research on this unique porous material, especially for the elimination of endocrine disrupting compounds (EDCs) from aqueous solutions via adsorption. Several controlling variables such as tunable porosity, hierarchical structure, immense pore volume and surface area, excellent adsorption and recyclability performances offer a new insight when compared with traditional adsorbents. The instability and toxicity of MOFs in water are a huge challenge in practical applications. Nevertheless, their capability and selectivity for the removal of EDCs can be justified by employing suitable metal ion clusters, organic linkers, and surface/bulk modifications. This review discusses the performance of several MOFs for the extermination of diverse categories of EDCs and the plausible adsorption mechanism involved. The importance of MOFs in environmental applications signifies that the development of advanced technology is required for the successful removal of EDCs from the aqueous environment.
\end{abstract}

Keyword: Metal-organic frameworks; Adsorption; Endocrine disrupting compounds; Water treatment 
